\title{
Margit Mayer \\ Städtische Bewegungen in den USA: \\ 'Gegenmacht' und Inkorporierung
}

Einschätzungen über die Rolle von städtischen Bewegungen für gegenwärtig in der BRD stattfindende Umstrukturierungsprozesse sind von unterschiedlichsten und bisweilen merkwürdig konvergierenden politischen Interessen geprägt. Nicht nur die Ökolibertären bei den GRÜNEN propagieren die Nutzbarmachung kleiner Netze und intermediärer Strukturen, auch innerhalb der CDU wird die Öffnung gegenüber »alternativer Basiskultur « und Selbsthilfeinitiativen gefordert. Diesen Interessen an einer neuen »Sozialgesellschaft « entspricht in SPD-Kreisen eine Aufwertung von alternativen ökonomischen Projekten im Rahmen von Ansätzen zu einer unkonventionellen Beschäftigungspolitik. Was jedoch die jeweiligen Verwirklichungschancen oder die faktischen sozialstaats- oder beschäftigungswirksamen Effekte solcher Einbindungsstrategien sein könnten, ist in der BRD - da die Wirkungen lokaler Modellprojekte nur mit Vorsicht generalisiert werden dürfen - noch schwer ausmachbar. Über den Status der veränderten städtischen Bewegungen und über ihre Rolle in gegenwärtig stattfindenden Restrukturierungsprozessen kann viel spekuliert und politisch gestritten werden, vor allem so lange einer verbreiteten Einbeziehung gesellschaftlicher Selbstorganisationspotentiale noch massive Barrieren und politische Vorbehalte entgegenstehen.

Ein Blick in die USA, wo gesellschaftliche und politische Strukturen eine relativ bruchlose Inkorporierung sozialer, v.a. städtischer Bewegungen in die auf Umbau des Wohlfahrtsstaats zielenden Strategien erlaubten, kann deshalb für die hier geführte Diskussion sehr nützlich sein. Die städtischen Bewegungen der USA haben sich während der letzten 20 Jahre grundlegend transformiert; das Terrain für politisches Handeln der Bewegungen hat sich - sowohl aufgrund zunehmender gesellschaftlicher Spaltungsprozesse als auch wegen der Inkorporierungsprozesse - verändert. Die Dimensionen eines verschobenen politischen Konfliktszenarios zeichnen sich deshalb in den USA deutlicher ab. Bei allen Unterschieden, sowohl was die Dynamik sozialer Bewegungen als auch den Kontext der politischen Kultur in den beiden Gesellschaften betrifft, können aus dieser Entwicklung wichtige Hinweise für die hiesige Auseinandersetzung gewonnen werden.

\section{Städtische Bewegungen: Von Protest zu Programm}

Während der 60er Jahre erfuhren die US-amerikanischen Metropolen eine Revolte, die verschiedentlich sogar als »städtische Revolution« bezeichnet wurde (Bell/Held 1969; Mollenkopf 1983). Im ganzen Land führten Stadtteilgruppen Kampagnen durch, um sich menschenwürdige Wohnverhältnisse zu erkämpfen, Kaputtsanierung zu verhindern, oder sich Einfluß in öffentlichen Einrichtungen zu verschaffen. Dabei wurden sowohl phantasievol- 
le wie militante Methoden eingesetzt, pragmatische Aktionen wie Demonstrationen oder Blockaden von Räumungsbaggern hatten genauso ihren Platz wie das Experimentieren mit »utopischen« Lebensformen. Bisweilen schienen sogar die in der amerikanischen Gesellschaft so tief verwurzelten Rassenschranken überwindbar, wo in »Urban Homesteading«Projekten genauso wie in öffentlichen Hearings Schwarz und Weiß neue Erfahrungen des Zusammenarbeitens machten. Diese Phase innovativer und kämpferischer Aktionen im Kontext der städtischen Krise hatte als Voraussetzung auch die Ghetto-Aufstände seit 1964; und sie entfaltete sich im Kontext einer umfassenden gesellschaftlichen Umbruchsituation: Die Bürgerrechtsbewegung, die entstehende Frauenbewegung, die Studenten- und Antivietnamkriegs-Bewegung beeinflußten den städtischen Protest genauso wie die Ausdehnung der amerikanischen Alternativkultur.

Resultat war jedenfalls eine starke und spürbar präsente städtische Bewegung, der es gelungen ist, unkonventionellen Protest als wirksames Mittel zur Realisierung ihrer Forderungen (»Protest as a political resource«, vgl. Lipsky 1968) einzusetzen. Eine Folge waren die relativ massiven staatlichen Zuwendungen für die marginalisierte städtische Bevölkerung (Präsident Johnsons »War against Poverty«), die auch eine wichtige Basis für die Konsolidierung des Protests wurden. Aus vielfältigen Quellen gespeist und vom Kontext der gegenkulturellen Mobilisierung der 60er durchwirkt, vermischte sich hier eine höchst heterogene Protestkultur zu dem, was Beobachter schließlich als »Neighborhood Movement « bezeichneten. Dies Neighborhood Movement machte auch während der 70er Jahre noch Schlagzeilen, obwohl die Akteure nicht mehr die Aufständischen der schwarzen Ghettos waren, sondern verschiedene »Citizen Groups«, die sich in unterschiedlichen Formen für ihre »Community《 engagieren (Boyte 1980; 1984). Neben traditionellem »Community Organizing« mobilisieren inzwischen auch die sog. »A ufwerter« für ihren Stadtteil: Der Übergang von alternativen Squattern zu Luxusmodernisierern war im rasanten Umstrukturierungsprozeß vieler Großstädte ganz fließend, vor allem weil auch letztere Gruppe durchaus aktiv für verbesserte städtische Dienstleistungen und mehr Mitspracherechte, aber auch für Umweltschutz und urbane Lebensqualität kämpft. Daneben sind nach wie vor eine Vielfalt von ethnischen und kulturellen Interessenorganisationen und politischen Gruppen Teil des Neighborhood Movement.

Diese verschiedenen Richtungen lassen sich analytisch auseinanderhalten; in der Realität existieren sie meist in Überlappungen und Überschneidungen. Es ist wichtig, die jeweiligen sozialen Träger und die Gründe für ihre Mobilisierung zu unterscheiden, um die sich herausbildenden neuen Konfliktlinien und die Chancen politischen Handelns unter Bedingungen zunehmender »Lokalisierung« und »Privatisierung« von gesellschaftlichen Auseinandersetzungen erkennen zu können. Es sind insbesondere vier Trends unterscheidbar, die vor dem Hintergrund städtischer Umstrukturierungsprozesse zu sehen sind.

1. Eine Richtung innerhalb der amerikanischen städtischen Bewegungen ist als defensive Reaktion etablierter Arbeiter-Quartiere auf einen »Angriff von außen«, auf den drohenden Verlust bestimmter Privilegien zu verstehen. Dieser Angriff kann sowohl die Form von Aufwertung (Gentrifizierung) als auch die von Verfall des Viertels annehmen. Typisch ist das stabile (ethnisch-) weiße Arbeiterviertel, mittlere Einkommensschichten, die sich in den alten industriellen Städten und Sektoren von Veränderungen ihrer Umgebung (d.h. häufig 
auch: der Veränderung der rassischen Zusammensetzung ihres Viertels) beeinträchtigt sehen. Das Ziel, ihr Viertel zu verteidigen und eigenständig zu kontrollieren, beinhaltet sowohl Opposition gegen staatliche Maßnahmen (die gewachsene Zusammenhänge zerstören) und gegen Banken und Firmen (die dem Stadtteil durch »Disinvestment « oder Deindustrialisierung schaden), als auch gegen die vermeintlichen Eindringlinge, wobei dann zuweilen die rassistischen Untertöne des amerikanischen Populismus zum Tragen kommen.

2. Die zweite Gruppe sind selbstorganisierte Initiativen in verslumenden, von Ausgrenzung bedrohten Stadtteilen, von marginalisierten und unorganisierten Bevölkerungsgruppen. Sie haben längst damit begonnen, ihre von Planern und Investoren benachteiligten oder von traditionellen politischen Institutionen ignorierten Interessen selbst in die Hand zu nehmen. Wo Verfall und Leerstand um sich griffen, wo Sozialprogramme gekürzt und gestrichen wurden, versuch(t)en diese Gruppen, auf möglichst pragmatische Weise das Überleben ihres Stadtteils zu sichern. Dazu nutzte man die geknüpften Verbindungen zu und Druckmöglichkeiten gegenüber der Stadtverwaltung genauso wie verschiedenste Formen von Selbsthilfe und eigene Stadtteilentwicklungsstrategien. Die Führung dieser Gruppen, die einen mobilisierten (oft schwarzen oder hispanischen) Stadtteil repräsentieren, wurde im Lauf der Zeit sehr häufig in die unteren Ränge des politischen Systems aufgenommen, wo sie in die Verwaltung der Programme, die sie ursprünglich kontrollieren wollten, eingebunden wurden. Gekoppelt mit der Suche nach Interessen auf dem freien Markt, die bei den Überlebensanstrengungen behilflich sein könnten, setzte sich diese Methode als gangbarste durch. Die Stadtteilgruppen wurden zu gemeinnützigen stadtteilbezogenen (Wohnungsbau- und Entwicklungs-) Gesellschaften und Dienstleistungsorganisationen.

3. In einem andern Teil der amerikanischen städtischen Bewegungen waren und sind die neuen Mittelklassen treibende gesellschaftliche Kraft und ausschlaggebender politischer Akteur. Als Produkt und Träger der Ausdehnung des tertiären Sektors (und dadurch ausgelöst des 'Back-to-the-City Movement') ist ihre Interessenlage von hohen Ansprüchen an »Lebensqualität « und urbaner Kultur bestimmt. Ihre Aktivitäten entzünden sich an traditionellen Defiziten in der amerikanischen Versorgung des Reproduktionsbereichs wie an zunehmenden Umweltproblemen, die in besonders krassem Maß in den »boomtowns« des »S Sunbelt« zum Ausbruch kommen (Houston, Dallas). Ihre Haltung zum Staat umfaßt die Bandbreite von traditionellen Forderungen nach mehr Einfluß auf die Kommunalregierung bis hin zu radikaldemokratischen Kommunalisierungsvorstellungen. Bisweilen finden bei dem Versuch, eine »gebrauchswert«-orientierte Haltung zur Stadt und zu städtischer Kultur durchzusetzen, bemerkenswerte Politisierungsprozesse statt. Genauso häufig jedoch führt die Voraussetzung zur Entfaltung des angestrebten Lebensstils, die Aufwertung und Attraktivierung des Viertels, aber auch zu Verdrängung und Marginalisierung minoritärer und armer Gruppen.

4. Schließlich sind diejenigen Teile der Neighborhood-Bewegung zu nennen, in denen das treibende Element von der Gefährdung der lokalen Kultur bzw. ihres Territoriums ausgeht. Sowohl Gruppen, deren Anliegen die Autonomisierung ethnischer Normen, als auch solche, die um autonome kulturelle Normen kämpfen, sind hier gemeint. Beispiel für erstere wäre die »Latino Community« in vielen amerikanischen Großstädten oder auch Harlem in New York; Beispiel für letztere die »Gay Community《 in San Francisco. Das heißt, Minori- 
täten, die in der Auseinandersetzung mit der hegemonischen Kultur ihre Identität zu finden und zu verteidigen suchen, organisieren sich dazu auf territorialer Basis, um ein größeres $\mathrm{Ma} ß$ an Sicherheit zu erreichen (vgl. St. Epstein 1987). Auch dieses Potential entwickelt sich in durchaus unterschiedliche Richtungen, zu »subkulturellen Stämmen« wie zu virulenten Herausforderungen für die städtische Administration.

5. Auch eine fünfte Kategorie, die jedoch nicht eigentlich einem soziostrukturellen Träger zuzuordnen ist, nämlich die überregional organisierten politischen Verbände, wäre zu nennen. Gruppen wie »Citizens Action«, ACORN (Association of Community Organizations for Reform Now) oder NPA (National People's Action) versuchen, auf überregionaler und nationaler Ebene die diversen Manifestationen des Neighborhood Protests zu koordinieren und zu verschärfen. Sie definieren sich als »multi-ethnisch « und »multi-issue«, d.h. Proble me des kollektiven Konsums (Steuern, Stromtarife, Gesundheitsversorgung, AKWs) werden genauso thematisiert wie Umwelt-Issues oder Einschränkungen von Bürgerrechten (Delgado 1986: 213). Als »soziale Bewegungs-Organisationen« (SMOs) reflektieren sie für die städtische und Community-Ebene das im amerikanischen Bewegungssektor vorherrschende Vertrauen in die Funktionstüchtigkeit der demokratischen Institutionen. Das Einklagen angemessener Dienstleistungen und die Verteidigung eines gewissen Lebensstandards sind hier eher Vehikel zur Erreichung des primären Ziels, »grassroots empowerment « und offene Partizipationskanäle herzustellen, damit das existierende Institutionengeflecht (wieder) im Interesse der Stadtteilbevölkerung funktioniert. Auch diese politischen Organisationen sind während der letzten Jahre zunehmend in die Interessengruppen-Politik eingestiegen, die kompetitiv und vornehmlich in der elektoralen Arena ausgetragen wird. Fast alle dieser Organisationen führen Fortbildungsprogramme für 'Führungskräfte' durch, wo Kenntnisse der »Mikro-Beziehungen der Macht, Umgang mit den Medilen, RessourcenEntwicklung, Verhandlungs-Qualifikationen und Kampagnen-Planung« vermittelt werden (Delgado 1986). Bis auf die für Wahlkampagnen nützlichen Fertigkeiten sind dies allesamt Qualifikationen, für die v.a. in der heute vorherrschenden Form von Stadtteilarbeit, der Community Development Corporatation (s.u.), großer Bedarf besteht. In einzelnen Organisationen steht auch Widerstand gegen drohende US-Intervention in Lateinamerika auf der Tagesordnung oder Haushaltskürzungen und Rüstungsausgaben. Dennoch muß man sehen, daß die »Organizers« immer weniger die ursprünglichen politischen Motivationen der Gründergeneration der Stadtteilaktivisten teilen.

Das Profil der amerikanischen städtischen Bewegungen hat sich im Verlauf der letzten Jahre unter dem Druck der ökonomischen Krise und unter dem Einfluß diverser lokal- und bundesstaatlicher Strategien gewandelt. Während die städtischen Bewegungen der 60er Jahre, egal welche räumliche Segregation sie verteidigten, sich in der Auseinandersetzung mit staatlichen Interventionen und privaten Investorinteressen häufig spontan politisierten, und während ihre Organisationen vor allem auf Konfrontation und Mobilisierung setzten, wird heute das Ziel (der Verbesserung der jeweiligen Lebenssituation) primär durch eigene Entwicklungsaktivitäten verfolgt. Wo vormals militante, konfrontationslustige Stadtteilgruppen Sit-ins oder Streiks inszenierten, öffentliche Hearings oder den geschäftsmäßigen Gang der Behörden störten - wie die Renegades in New York oder die Commandos in Milwaukee - haben wir längst stadtteilverankerte Entwicklungsgesellschaften, Ausbildungs- 
und Arbeitsbeschaffungsprojekte, Drogenprojekte, und andere auf Feinsteuerung beruhende Strategien zur Revitalisierung city-naher Wohngebiete. Die Karriere von vielen solcher Stadtteilgruppen wird in einem Bild (New York Times, 5. Dez. 1981) eingefangen: Der Geschäftsführer der Commandos, »a school drop-out (d.h. ohne Schulabschluß) und ehemaliger Straftäter ... trägt heute einen Aktenkoffer, verhandelt Verträge und sitzt hinter einem polierten Nußbaumschreibtisch. In der Nähe steht die schwarze Fahne mit der goldenen geballten Faust, das Symbol der Commandos« (ibid, Übersetzung M. M.). Eine andere Beobachtung der New York Times, die den subtilen Veränderungsprozessen immer eng auf der Spur blieb: »Die Stadtteilgruppen, die früher im ganzen Land verbesserte Dienstleistungen von den Rathäusern forderten, erbringen diese jetzt selbst«, meldete die New York Times 1983 (John Herbers, »Cities turn to Private Groups to Administer Local Services«, 23.5. 1983): Ob sie Straßen reparieren oder Gehsteige bauen, Sozialstationen managen oder Obdachlosenayle betreiben, Parks unterhalten, Wohnungen renovieren oder Behinderten helfen - »solche Herunterzonungen von staatlicher Kompetenz - unvorstellbar in der Vergangenheit - schreiben das jüngste Kapitel der rapide sich entwickelnden Rolle von Stadtteilgruppen und 'Privatisierung' der lokalen Regierungen« (ibid). Diese Beschreibungen unterscheiden nicht zwischen den unterschiedlichen Ausformungen und Funktionen, die einer Community-based Organization (CBO) - je nach ihrer Lage - zukommen. Es macht jedoch einen Unterschied ob die Organisation in einem revitalisierenden Arbeiterviertel, im ausgegrenzten Ghetto oder in einer gentrifizierenden Lower East Side angesiedelt ist. Insgesamt jedoch gilt, daß das Bild des sozialen Bewegungssektors in amerikanischen Städten heute, im Gegensatz zu den 60er Jahren, vor allem von drei Kennzeichen geprägt ist:

1. Beobachter konstatieren eine »bewegungsarme« Situation. Lediglich punktuelle Protestaktivitäten um Bodennutzungs- und Wohnungskonflikte seien entflammt, z.B. bei der Erneuerung von Times Square in der Stadt New York, oder ähnlichen, wenn auch nicht ganz so protzigen, Ausdehnungs- und Aufmotzungsprozessen der zentralen Geschäftsbereiche in den Großstädten, die sog. »global city«-Funktionen (und damit unternehmensbezogene Dienstleistungen) an sich ziehen konnten. Alles was die Clinton Coalition of Concern (die sich gegen das Stadterneuerungsprojekt am Times Square organisierte) herausschlagen konnte, waren einige Garantien furr »low-income housing « und »small business«, in anderen Fällen führt der Druck auf die lokalen Politiker und Institutionen bisweilen auch zu weitergehenden Konzessionen an die urbane Lebensqualität und Auflagen für die großen Entwicklungsgesellschaften, Beiträge für Verbesserungen in ärmeren Stadtteilen abzuzweigen (vgl. die sog. Linkage-Programs in San Francisco oder Boston, Keating 1986).

2. Die vorherrschende Form von Stadtteilaktivität in den 80er Jahren ist »development", d.h. die Herstellung oder Erneuerung von Gebäuden und/oder gewerblichen Unternehmungen. »Development« setzt jedoch, im Gegensatz zum klassischen Community-Organizing nach dem Alinsky-Modell, erhebliche Kapitalressourcen, ausgebildetes Personal und technisches und planerisches Kow-how voraus. Um an diese nötigen Ressourcen zu gelangen, müssen die Gruppen ins Kreditgeschäft einsteigen, und zu diesem Zweck ihre technische Kompetenz und Zuverlässigkeit unter Beweis stellen. Sie werden in dieser Hinsicht nie mit Kapitalgesellschaften konkurrieren. Viel wichtiger ist deshalb der Eindruck, daß eine 
CBO (und nur sie) den politischen Willen ihrer Basis repräsentiert und diesen falls nötig mobilisieren kann. Das kompensiert für mangelndes Kapital und einschlägige Erfahrung (vgl. Kolodny 1985: 16). Gefragt ist also ein Balanceakt zwischen institutionellem und praktischem Erfolg (als Entwicklungsgesellschaft) und politischer Vertretung, die imstande ist, sensibel die Interessen ihrer Basis aufzugreifen. Dazu muß die Organisation ihre aktivistische, basisorientierte Dimension unbedingt beibehalten (Gardner 1983; Friedman/Schweke 1981).

3. Das dritte Merkmal heutiger Stadtteilaktivität ist ihre Beteiligung an Implementationsfunktionen städtischer Dienstleistungen. Auch hier konvergierte die zunächst aus den staatlich und ökonomisch induzierten Verfalls- und Ausgrenzungsprozessen geborene Selbsthilfe-Aktivität, mit der man bspw. durch Besetzung und Renovierung leerstehender Häuser und Verwandlung von Schrotthalden in blühende Gärten - zunächst durchaus gegen herrschendes Recht und Eigentum - Fakten schuf, mit schnell Schule machenden lokalpolitischen Strategien zur Lösung der kommunalen Finanz- und Integrationskrise. Die Beispiele aus dem zitierten NYT-Artikel sind nur ein kleiner Ausschnitt für die vielfältigen (lokal)staatlichen Dienstleistungen, deren Erbringung inzwischen abgewälzt wird auf gemeinnützige und basisnahe Stadtteilorganisationen.

Ein Resultat des breiten Neighborhood Movement der 60er und 70er Jahre ist also, daß eine Reihe von Forderungen der städtischen Bewegungen mit Konzessionen erfüllt, mit intensivierten Formen von Bürger- und Betroffenenbeteiligung institutionalisiert und durch Übertragung von Implementationsfunktionen in die Kompetenz der 'Betroffenen' gestellt wurden. In diesem Prozeß hat sich nicht nur die Funktion städtischerSelbstorganisation gewandelt (und zwar nicht unbeeinflußt von den wechselnden staatlichen Strategien); sie spiegelt inzwischen auch die ihr zugrundeliegende gesellschaftliche Polarisierung, die während der letzten 15 Jahre die Stadtentwicklung erfaßt hat. Stadtteilaktivität als (vornehmlich mittelklasse-geprägte) Citizen Action tritt in den Hintergrund; sie konzentriert sich zusehends mehr im Bereich der gesellschaftlich Marginalisierten (ungeschützte Arbeitsverhältnisse, Informalisierung) einerseits und der an Lebensqualität orientierten, tertiären oder in der aufgewerteten Produktion beschäftigten Yuppies andererseits. Je nachdem ob die Community-Development Corporation (CDC), die »alternative service delivery organization« oder auch die punktuelle 'Kampforganisation' sich auf die Ressourcen und Potentiale der ersteren oder letzteren Gruppe bezieht, entwickelt sie sich nach recht unterschiedlichen Gesetzmäßigkeiten und innerhalb unterschiedlicher Handlungszwänge. - Besonders deutlich läßt sich diese Unterschiedlichkeit am Beispiel der krass polarisierten 'Boomtowns' à la Houston illustrieren: Wo die Organisationen der hispanischen, schwarzen und neuen Immigrantenbevölkerung seit Jahren ohnmächtig den Zumutungen einer ohne alle Umweltoder soziale Auflagen expandierenden Privatindustrie ausgeliefert war, führen erst die Auswirkungen der »Externalisierungen« auf die Lebensqualität der hochbezahlten Angestellten der multinationalen Konzerne, und deren Organisationen, die sowohl Ressourcen als auch politischen Einfluß mobilisieren können, zu sichtbaren Konflikten, Öffentlichkeit, und Ansätzen von Abhilfe (vgl. Feagin 1985; Fisher 1987). 


\section{Staatliche Inkorporierungsstrategien und -formen}

Auf lokaler Ebene wird bereits seit den frühen 70er Jahren mit sozial innovativen, auch alternativen Mechanismen und Verhandlungsformen, und mit Einbeziehung neuer basisnaher Trägerorganisationen experimentiert. Diese hatten schließlich Qualitäten ganz eigener Art unter Beweis gestellt; nämlich dort, wo es ihnen gelang, sterbende Stadtteile zu revitalisieren, indem sie - durch Mobilisierung lokaler und ethnischer Bindungen, durch erkämpfte Subventionen aus verschiedenen Bundes- und Kommunalmitteln und Geldern von Stiftungen, Banken und Privatunternehmen - eigene Ausbildungs- und ArbeitsbeschaffungsProgramme zusammenstellten, und diese häufig auch mit eigenen Wohnraumsanierungsund Stadterneuerungsprojekten verknüpften. Das heißt natürlich nicht, daß die städtische Verwaltung in einem Akt weitsichtiger Planung solche Selbsthilfe-Ansätze koordiniert aufgegriffen hätte. Die städtischen Programme und Modelle, die bspw. in New York aufgelegt wurden (New York spielte während der 70er Jahre eine Vorreiterrolle in dieser Hinsicht), waren zunächst eine disparate und unkoordinierte Reaktion auf den Druck der Mietergruppen und Community-Organisationen einerseits, und auf die Finanzkrise, das Sanierungsproblem verrotteter Stadtteile, und das Problem sozialer Integration von Minoritäten und Armutsbevölkerung andererseits. Das Community Management Program (1972) und das Sweat Equity Homesteading wurden erst 1978 in einer eigenen Verwaltungseinheit (Division of Alternative Mangement Programs) innerhalb des Amts für Stadterneuerung koordiniert. Die verschiedenen DAMP-Programme zeichnen sich dadurch aus, daß sie Eigenarbeit der Mieter voraussetzen. Auswertungen der Modellprojekte zeigten bald, daß sowohl verbesserte Mietzahlungsraten als auch die Frequenz der Privatisierungen für den »Erfolg « der Experimente im Sinne der Stadtverwaltung sprachen. Die städtische Förderung von Selbsthilfe und Selbstorganisation in New Yorker Stadtteilen erwies sich zweifelsfrei als "anspruchssenkend (Katz 1987).

Der Transformationsprozeß des 'Neighborhood Movement' fand aber nicht nur lokal, in Interaktion mit örtlichen Programmen statt. Er ist viel genereller eine Reaktion auf die veränderte ökonomische Situation (seit der Krise Mitte der 70er Jahre) und auf die staatliche Wende zu einer neuen konservativen Austeritätspolitik. Neben Ausgabenkürzungen, Privatisierung und Abbau sozialer Dienste besteht der neue Regulationsmodus auch aus der subsidiären Mobilisierung gesellschaftlicher Eigenarbeit und kleinunternehmerischer Innovationspotentiale. Auf lokaler Ebene wurden diese Potentiale flexibel aufgegriffen. Das amerikanische Institutionengefüge (mit seiner offenen und fragmentierten Struktur) und die politische Tradition der USA ermöglichen nicht nur das Eingliedern vormals ausgeschlossener Gruppen in politische Repräsentations- und Verhandlungsstrukturen, sondern auch, deren Organisationen für die Implementation bestimmter Programme und Dienstleistungen in städtischen Vierteln zu engagieren. Diese Indienstnahme »gesellschaftlicher Selbstorganisation « erreichte allerdings im Kontext des Reaganschen »New Voluntarism« eine qualitativ neue Ebene.

Die jeweiligen bundesstaatlichen Maßnahmen strukturieren das Terrain, in dem die städtischen Organisationen operieren. So bewirkten die Mittel und Programme der 60er Jahre einerseits Sanktionierung und Legitimierung der stadtteilbezogenen Interessenvertretung 
(und dabei auch die Herausbildung gemeinsamer Handlungsebenen zwischen schwarzen Gruppen und eher mittelständischen »Citizens Organizations《), sie induzierten andererseits aber auch eine $A$ bhängigkeit der Gruppen von staatlichen Zuwendungen. Intensiviert wurde diese Abhängigkeit durch die (unter der Carter Administration) vom Office of Neighborhood Development verteilten Gelder, mit denen Programmentwicklung, Verwaltung und Gehälter der Organisationen subventioniert wurden. In Carter's Urban Policy wurden die Probleme der Stadtteile (erstmals seit dem Model Cities Act von 1966) wieder zum Objekt nationalstaatlicher Politik gemacht. Die lokale Gemeinde und ihre Stadtteil-Organisationen wurden zum »Dritten Partner« in den städtischen Revitalisierungsbemühungen erklärt. Mit der Gründung der National Neighborhood Commission konnte sich die Stadtteilbewegung sogar mit personellen Verflechtungen einen Stützpunkt innerhalb der Staatsbürokratie (im Ministerium für Wohnungswesen und Stadtentwicklung) errichten. Diese systematischen Konzessionen an die Stadtteilbevölkerung (wenn auch bescheiden im Vergleich zu den Milliarden, die unter den Model Cities und Community Action Programmen der $60 \mathrm{er}$ Jahre autorisiert waren) verfolgten vor aliem den Zweck, private Investitionen $\mathrm{zu}$ veranlassen, Privatisierungsprozesse mit Hilfe staatlicher Instrumentarien zu effektivieren und den privaten Sektor systematisch an der städtischen Wiederbelebung zu beteiligen (v.a. UDAG, vgl. Gatons/Brintnall 1985).

Resultat dieses Prozesses waren stabile Ansprechpartner auf der lokalen Ebene, die

- formell und informell an Verhandlungsstrukturen und legislativen Prozessen beteiligt wurden,

- zum unverzichtbaren Partner in sog. Public/Private Partnerships wurden,

- und eine Rolle bei der Implementation gewisser öffentlicher Dienstleistungen übernahmen.

Nachdem diese Entwicklung in die Wege geleitet war, bedeutete die Reduktion bzw. Eliminierung von Förderprogrammen für Stadtteil- und marginalisierte Interessen, wie sie 1981 mit der Reagan-Administration einsetzte, keineswegs das Ende dieser Formen routinisierter Repräsentation und Einbindung lokaler Interessen, aber sie transformierte ihre Funktion.

Zwar zielte schon die Cartersche 'Neighborhood Policy' primär auf die Mobilisierung des Privatsektors für die städtische Revitalisierung, wobei Community Organizations als relevante Vehikel angesehen wurden. Aber solange mit Hilfe staatlicher Mittel noch Rechte der Neighborhoods erkämpft und gewährleistet werden konnten (bspw. das Recht, bei der Verteilung von CDBG-Geldern [Globalzuweisungen für Stadtteilentwicklung] Betroffenen-Interessen geltend zu machen, oder auch das Recht, selbst ein Instandsetzungsprojekt durchzuführen), bestand für die Community Organization noch ein Zusammenhang zwischen politischem Mobilisierungspotential und eigener Programmaktivität. Sobald jedoch (unter der Reagan-Administration) staatliche Mittel nur noch für spezifische Projekte ${ }^{1}$ und auf bestimmte Art erhältlich wurden, entwickelten sich die Gruppen zunehmend zu (Co-) Produzenten/Verwaltern öffentlicher Güter und Dienstleistungen. Wie unter früheren Republikanischen Administrationen auch, wird bundesstaatliche Einmischung in lokale Marktprozesse seit 1981 wieder abgelehnt. Städtische Politik seit Reagan kennt also kaum explizit auf Städte bezogene Programme; sie setzt sich vielmehr zusammen aus den Wirkungen von Steuerkürzungen, Erhöhung der Militärausgaben und Streichungen bei den Sozial- 
ausgaben (Glickman 1984). Mit dieser Kombination und mit dem »New Federalism« (also der Herunterzonung von staatlicher Entscheidungsbefugnis auf einzelstaatliche, regionale und lokale Ebenen) und dem »New Voluntarism《 (der Delegation staatlicher Kompetenzen an soziale Organisationen) intervenierte die bundesstaatliche Administration sehr wohl und mit folgenschweren Effekten für die Städte und Stadtteile.

Der Neue Föderalismus, der auf gesteigerte Flexibilität der Lokalregierungen zielt, machte die Einzelstaaten für die Erbringung bestimmter Dienstleistungen verantwortlich, senkte die Bundesausgaben für Reformprogramme und ersetzte zweckgebundene Zuweisungen durch »Block Grants«, deren Verteilung in das Ermessen der Lokalregierung gestellt wurde (was unter den durchschnittlichen lokalen Bedingungen bedeutete, daß sich der Anteil für Stadtteile stark verringerte, vgl. Petersen 1984). Steuerlichen Anreizen kommt für die Stadtentwicklungs- und Wohnungspolitik eine größere Rolle zu als je zuvor. Zusätzlich ermöglichen die im Economic Recovery and Tax Act (ERTA, 1981) beschlossenen Maßnahmen und Steuerkürzungen vor allem den großen Unternehmen erhöhte Mobilität: Standortwechsel (in Niedriglohngebiete und solche mit niedrigem gewerkschaftlichen Organisationsgrad) wurden erleichtert, bereits existierende Markttendenzen also verschärft. Unter den auch nominal auf die Stadtentwicklung bezogenen Programmvorschlägen sind die »Unternehmenszonen« zentral. Dies Konzept sieht vor, daß in (vom Bundesministerium für Wohnungswesen auszuwählenden) Stadtteilen Steuern und Auflagen reduziert und Investitionssteuervergünstigungen für Wohnungsbau und -modernisierung erlassen werden, die Kapitalertragssteuer eliminiert und andere Anreize mehr geboten werden, damit Firmen sich dort ansiedeln (Butler 1981; Bendick/Rasmussen 1986).

Neben Modifikationen der CDBG- und anderer Programme aus der Carter-Ära, die vor allem auf eine finanzielle Benachteiligung der Stadtteile hinausliefen, beteiligte sich die Bundesregierung mit einer Reihe von Modellprojekten an der Suche nach gangbaren tripartistischen Arrangements. Eine Reihe von Pilotprogrammen explorieren, in welchem Maß der Staat eine Rolle spielen kann bei der Umstrukturierung bestimmter Arbeitsmärkte und Produktionsweisen - sei's durch die Herstellung prekärer, ungeschützter Arbeitsplätze, oder durch die Herunterzonung und Delegation bestimmter staatlicher Kompetenzen. Neben dem erwähnten Enterprise Zones Program setzen auch die Alternatives to Service Delivery im Stadtteil verankerte Organisationen (CBOs) als notwendiges Element voraus.

Ein 1982 verabschiedetes Programm Partnerships for Service Delivery fordert Nachbarschaftsorganisationen auf, »kreative und innovative« Arrangements zur Erbringung und Organisation von Dienstleistungen in allen möglichen kommunalen Bereichen (Umwelt, Verbrechensbekämpfung, Gesundheit, Erziehung, Freizeit etc.) zu entwickeln. Diese Bereiche zeichnen sich dadurch aus, daß Dezentralität in der Erbringung der Dienstleistung effizienter ist, und daß die Interaktionsbeziehung zwischen »Konsumenten《 und »Produzenten« ein bestimmtes Maß an Sensibilität erfordert. Ein anderes Beispiel sind die sog. Community Development Partnerships: Sie sind Auszeichnungen für solche Stadtteilgruppen, denen es gelang, den lokalen Einsatz von CDBG-Mitteln mit einem besonders hohen Anteil von Privatinvestitionen zu verknüpfen (so daß also bspw. ein $1 \mathrm{CDBG}$ - 13 private $\$$ locker machte). Eine der wenigen Stadtteil-Einrichtungen, die noch direkt von der Bundesregierung finanziell unterstützt wird, ist das National Center for Neighborhood Enterprise, 
das die Fähigkeit von Stadtteilgruppen, unternehmerisch tätig zu werden, erforscht, erprobt und propagiert. Hier werden die »Mediating Structures«-Konzepte des American Enterprise Institute angewandt, um die Rolle von Stadtteilorganisationen bei der Lösung städtischer ökonomischer und sozialer Probleme zu testen. Die propagierte Lösung heißt: "Ineffektive und veraltete soziale Wohlfahrtsprogramme« sind durch neue Formen marginalisierter Industrien zu ersetzen (Berger/Neuhaus 1981; Woodson 1982, 1983). Beibehalten wurden Programme (wie Neighborhood Housing Services), die die Kooperation zwischen Privatfirmen, Banken, der Kommune und Stadtteilorganisationen bei der Reinvestition in verfallenen Stadtteilen fördern.

Neben Kürzungen und Umgewichtungen hat auch die Art und Weise, in der die verbleibenden staatlichen Zuwendungen an die Gruppen gelangen, Auswirkungen auf deren Handlungsspielräume und Handlungsweise. So stieg der Anteil der durch Steuerbefreiung und Suspendierung von Regulierungen zustandegekommenen Unterstützung. Als Folge orientierten sich die Stadtteilgruppen hin zu Aktivitäten, die mit Steuereinsparungen und Kreditgarantien »belohnt « werden (Clarke 1984). Je effizienter die Gruppen in der Auswahl der Projekte und in der Verhandlung ihrer Geschäfte sind, umso größer ihre Chance, ein eigenes Einkommen zu generieren. Die Anwendung komplizierter, technischer steuerlicher Prozeduren (in der Stadtteilrevitalisierung) hat allerdings eine Veränderung der Politikform zur Folge: Öffentliche Auseinandersetzungen (über die Verteilung direkter Zuschüsse) entfallen, »unsichtbare« Vergünstigungen wie Steuerkredite eignen sich nicht für Mobilisierungsprozesse.

Schließlich testen noch eine Reihe von Demonstrations-Projekten auf Bundesebene, mit welchen Arrangements die größtmöglichen Anteile von Eigenarbeit und lokalen Ressourcen involviert werden können. Für das seit 1985 laufende Neighborhood Development Demonstration Program bewarben sich über 300 Neighborhood-Organisationen, von denen 44 ausgewählt wurden. Auch hier ist die Voraussetzung für den Bundes-Zuschuß (von maximal $50000 \$$ für maximal $1 \mathrm{Jahr}$ ) das Auftreiben sogenannter »Matching Funds«, also Mitteln von Privatpersonen, Firmen, Geschäften oder sonstigen vor Ort angesiedelten Institutionen. Förderungswürdige Aktivitäten umfassen lokale Beschäftigungsinitiativen, Gründung von gewerblichen Betrieben, Erneuerung von Bausubstanz, Förderung und Finanzierung von freiwilligen Stadtteilerneuerungsinitiativen. Die penible Auswahl der Gruppen (sie müssen bereits 3 Jahre erfolgreich tätig sein, in ihrem Aufsichtsrat müssen mindestens $51 \%$ Stadtteilvertreter sein) und die Art der Zahlung (nicht nur die Verknüpfung mit vor Ort mobilisierten Mitteln, sondern auch die vierteljährliche Zahlungsweise nach Eingang von Leistungs- und Finanzbericht) gewährleisten, daß nicht unbestimmte stadtteilorientierte Selbstorganisation gefördert wird, sondern

- Planungs- und 'Fundraising'-Kompetenz in ganz bestimmter Form,

- Personalmanagementkapazitäten, um Angestellte und ehrenamtliche Mitarbeiter effizient zu koordinieren,

- Finanzierungs- und juristische Qualifikationen, um Tochtergesellschaften zu verwalten, und

- ein entsprechend ausgebildetes Personal, das jedes Programm auch implementieren kann. 
Weitere Demonstrationsprojekte sind unter dem Namen Quality of Life Initiatives vom Ministerium für Wohnungswesen und Stadtentwicklung lanciert worden: Im National Self-Sufficiency Project (1984) wurden 35 Mio \$ bereitgestellt, um lokale Public/Private Partnerships für eine besonders bedürftige Bevölkerungsgruppe, alleinerziehende Eltern (also meistens arbeitslose Mütter), nutzbar zu machen. Das Programm richtet sich an »hoch-motivierte« Alleinerzieher, um ihnen den Übergang von der Sozialhilfe-Abhängigkeit zu produktiver Beschäftigung zu erleichtern. Auch Arbeitsbeschaffungsmaßnahmen für arbeitslose Jugendliche werden im lokalen Partnerschafts-Verfahren getestet: Die seit 1983 laufende Minority Youth Training Initiative kombiniert die Ausbildung Jugendlicher in der Wohnungsinstandhaltung und -bewirtschaftung mit anschließender Arbeitsbeschaffung unter der Ägide einer Partnerschaft, die aus Bürgermeister, Sozialer Wohnungsbaubehörde und Privatsektor besteht. Obwohl die geringen Mittel, die in diesen Modellprojekten ausgegeben werden, auf den niedrigen Stellenwert verweisen, den solche Programme (im Vergleich zu früheren) einnehmen, zeigt jedenfalls die Tatsache, daß nur noch und ausschließlich die Entwicklung solcher Neighborhood Development Organizations gefördert wird, die bestimmte Kriterien erfüllen, den Trend auf: Aktive, gemeindebezogene Interessenvertretung wird sowohl gefördert und in Verhandlungszusammenhängen vorausgesetzt, als auch kanalisiert in der Weise, daß sie sich der ökonomischen Norm der Public-Private Partnership anpassen muß.

Gleichzeitig findet die Formierung von Stadtteilinteressen auch im Kontext einer breiten ideologischen Mobilisierung statt, die allem Anschein nach mit Erfolg private Firmen von den Vorteilen einer sozialen Definition des Profits, und davon »Philantrophie als Investition« zu betreiben, überzeugt hat (President's Task Force on Private Sector Initiatives 1984; Fosler/Berger 1982). Einzelne Konzerne (wie Control Data oder Xerox Co.) beteiligen sich finanziell und personell an tripartistischen Arrangements innerhalb der lokalen Gemeinde. Daneben haben sich verschiedene Vermittler- und Anlageberatungsorganisationen gebildet, um die von Privatkapital zur Verfügung gestellten Mittel zu koordinieren und ihren Einsatz zu effektivieren. Parallel zu dieser vermittelten Vergabe von Darlehen und Zuschüssen an die »fortgeschrittensten « gemeindebezogenen Selbsthilfe-Organisationen wird »begleitend erforscht« (z.B. von LISC, der von der Ford-Stiftung gegründeten Local Initiatives Support Corporation), wie eine bundesweite intermediäre Organisation Konzerne dazu bewegen könnte, bereitwilliger in gemeindebezogene wirtschaftliche Entwicklung zu investieren (Vidal u.a. 1985). LISC-subventionierte Projekte komplementieren die aktuelle staatliche Strategie, indem sie auf die »Urban Underclass« zielen, die aus der 'National Urban Policy' ausgegrenzt wurde. Staatlich verordnete »Trickle-down Austerity«, so wird hier argumentiert, könne die Problemkonstellation einer South Bronx nicht lösen — 》Self-help Community Development« sei die einzige effektive Mikrostrategie zur Behebung der sich in solchen Problemzonen manifestierenden Strukturprobleme (Sviridoff 1982).

Zusammengefaßt läßt sich also festhalten: Alle Bundesprogramme (UDAG, JIPA, CDBG), genauso wie eine Reihe von Einzelstaatsprogrammen setzen voraus, fördern oder belohnen die Beteiligung privater Firmen und Dienstleistungsunternehmen an der Produktion öffentlicher Güter. Neben den staatlichen Anreizen haben einige Konzerne auch eigene 
Gründe, sich in solchen Partnerschaften zu engagieren: Große Arbeitgeber innerhalb einer bestimmten Region bspw. müssen angesichts gegenwärtiger städtischer und ArbeitsmarktUmstrukturierungen sicherstellen, daß funktionale Reproduktionsmöglichkeiten für ihre Angestellten existieren, denn eine umfassende Bau- und Raumplanung wird weder zentral gesteuert noch unterstützt. Der zweite Partner in diesen Public/Private Partnerships, die Kommune, profitiert auch - obwohl sie weder gleichberechtigt noch gleich mächtig neben dem überlokalen Kapital steht - von der Partnerschaft, insofern diese ihr erhöhte Flexibilität und Innovationskapazität bei der Bewältigung von Stadtteilentwicklungsproblemen ermöglicht. Unentbehrlich ist der dritte Partner, die jeweilige Form von Stadtteilrepräsentanz, weil ihr Wissen und ihre Glaubwürdigkeit im Viertel Voraussetzungen für die Durchführung lokaler Projekte sind. Viele Stadtteilorganisationen begrüßen die Public/Private Partnerships und ihre eigene Rolle darin, weil sie Arbeitsplätze - z.B. für »aufstrebende Mitglieder der Underclass« - erschließen (Cunningham/Kotler 1983: 185; vgl. für viele weitere Beispiele Citizen Participation, 6/1, 1986). Aber nicht alle Stadtteilgruppen und ihre Zusammenschlüsse favorisieren so gradlinig die Ziele der Public/Private Partnerships. Trotz Kritik können sie jedoch mit ihrer Lobby-Praxis in Washington (z.B. National Low Income Housing Coalition) den Sog nicht aufhalten, in den ihre Basis vor Ort gerät. Während sie in Washington »demokratischere« Gegenvorschläge unterbreiten, konsolidiert sich in den Regionen die organisatorische, politische und finanzielle Infrastruktur der Public/Private Partnership. Ihre Präsenz führte faktisch zur Marktanbindung der Gruppen entweder in der Form, daß private Kreditgeber und Investoren die Führung übernehmen, oder in der Form, daß noch brachliegende Eigenressourcen (an Arbeitskraft, Ersparnissen oder Solidargemeinschaft) »kapitalisiert« werden.

\section{Die Rolle von Community-Based Organizations (CBOs) in der gespaltenen Gesellschaft}

Die beschriebẻnen Tendenzen von Einbeziehung der Protestbewegungen sind offensichtlich Teil einer umfassenderen staatlichen Strategie, die die Revitalisierung von Marktkräften in bislang ausgesparten oder vernachiässigten Bereichen als gesellschaftliches Steuerungsmittel einsetzt. Das Resultat für die Bewegungen ist ambivalent, denn die Einbeziehung bewirkt einerseits soziale Integration auf niedrigerem und konkurrenzbetonten Niveau; aber sie bietet andererseits auch Chancen, weil aktive, stadtteilverankerte Selbstorganisation nun vorausgesetzt und sanktioniert ist. Community Organizations geraten dabei wohl in die Rolle lokaler Selbstregulierungsinstanzen, die eingebettet sind in die umfassende Austerity- und ökonomische Restrukturierungspolitik. Aber diese neue Rolle bedeutet nicht gleich »gelungene Integration«, sondern zunächst einmal nichts anderes als eine Verschiebung des politischen Konfliktszenarios, die von den Beteiligten noch gar nicht in vollem Umfang wahrgenommen wird.

Die vielfältigen Segmentierungen und Polarisierungen in der heutigen Gesellschaft können nicht mehr mit den formalen Great Society-Programmen bearbeitet werden. Die sich herausbildenden neuen Formen staatlicher Intervention sind jedoch nur dann effektiv, wenn sie auf die Mitwirkungsbereitschaft dezentraler Organisationen setzen können. Neighbor- 
hood Organisationen, die die Interessen marginalisierter und/oder oppositioneller städtischer Gruppen politisch repräsentieren, werden heute angehört bei der Politikformulierung und wirken mit bei der Implementierung sie betreffender Programme - vor allem weil sie spezifisch vor Ort verankert sind. Diese politische Aufwertung der dezentralen Interessenorganisationen - die ja auch die Gefahren überschießender Partizipationspotentiale und abnehmender Berechenbarkeit birgt - wird konterkariert durch die privatwirtschaftliche Ökonomisierung, in die alle Gruppen (die darauf angewiesen sind) gedrängt werden.

Neighborhood Organisationen in den ausgegrenzten Stadtteilen sind scheinbar »darauf angewiesen«, denn viele, vor allem aber ihre Beratungs- und Betreuungsorganisationen, beteiligen sich aktiv an der Entwicklung weg von sozialstaatlicher Unterstützung hin zu gesellschaftlicher Eigenarbeit und kleinunternehmerischer bzw. informeller Innovationspotentiale. Sie unterstützen auch die Gesetzentwürfe, die die Verwendung von staatlichen Transferzahlungen an Arbeitslose (Arbeitslosenversicherung, Aid to Families with Dependant Children, General Assistance) für Betriebsgründungen erlauben würden. Sie sehen, schulen und vermarkten die besonderen Fähigkeiten der CBOs, wo es um die Mobilisierung von Eigenaktivität und die Schaffung von flexiblen Arbeitsplätzen in Bereichen marginaler Produktion geht. Manche profilieren sich auch durch ihre Fähigkeit, die Widerstände in der marginalisierten Bevölkerung, sich dem Arbeitsmarkt zur Verfügung zu stellen, kleinzuarbeiten. So ist es z.B. ein Ziel des 'Training Program' des National Congress of Neighborhood Women, schwarze 'Welfare Mothers' zu überzeugen, einen unterbezahlten und prekären Arbeitsplatz anzunehmen.

Das Development Training Institute dagegen favorisiert das Genossenschaftsmodell, weil hier (teures) Kapital durch (billige) Arbeitskräfte ersetzt werden könne, und »niedrige Löhne im Tausch für die Beteiligung am Wachstum und Wert der Firma« gern hingenommen würden (McNeely 1985: 9). Diese Beratungsorganisation schlägt, wie andere auch, den Gruppen die Ausweitung der »informellen und Heimarbeit« vor mit dem Argument, daß wein riesiger potentieller Abnehmermarkt existiere in den vielen jungen, wohlhabenden innerstädtischen Angestellten (...) die meist nicht die Zeit haben, solche Dienstleistungen für sich selbst zu erbringen« (ebd.). Solche Vorstellungen passen sich nahtlos ein in die gespaltene Stadt, wo Schattenwirtschaft ein expansiver Sektor für die steigende Nachfrage nach arbeitsintensiven Dienștleistungen geworden ist. Der "Yuppifizierung« einerseits entspricht so die gestiegene Nachfrage nach »vergesellschafteten Dienerinnen und Dienern«, wie Gorz die schlecht bezahlten 'Nicht-Arbeitsplatz-Besitzer' genannt hat (Gorz 1985).

Wo die Gruppen der staatlich definierten Rolle entsprechen, wirken sie mit an der Herstellung eines neuen Regulationsmodells (und tragen so die fortschreitende Durchstaatlichung der Gesellschaft ein Stück weiter). Dies Regulationsmodell bindet Wirtschaftsförderung und Sozialpolitik prozessual an »Betroffeneninteressen« an und verkoppelt damit die Rücknahme staatlicher Leistungen mit privatwirtschaftlicher Ökonomisierung und politischer Neutralisierung virulenter gesellschaftlicher Interessen. Es basiert also auf einem keineswegs widerspruchsfreien Balanceakt heterogenisierter gesellschaftlicher Interessen, in dem die Entfaltung autonomer und demokratisierender Potentiale weder durch die Marktanbindung noch durch die staatliche Norm völlig reguliert werden kann. Es hängt nach wie 
vor von den politischen Zielen, konkreten Utopien und den Ressourcen, die die Bewegungen mobilisieren können, ab, mit welchem Erfolg sich in diesem neustrukturierten Feld Politik machen läßt.

\section{"A Tale of Two Cities«}

Die amerikanischen Städte waren in den letzten 20 Jahren Schauplätze rasanter Verschiebungen. Während der 60er Jahre erschien die »Krise der Stadt« als Abwanderung einkommensstarker weißer Mittelschichten in die 'Suburbs', als Kapitalflucht und Desinvestition ganzer Branchen und als Aufstände innerstädtischer Bevölkerungsgruppen in den Slums der Großstädte. Während der 70er Jahre setzte die 'Finanzkrise' den verschiedenen Abwanderungs- und Erosionsprozessen der Sektoren, die vormals wirtschaftliches Wachstum und städtische Einnahmen bedeutet hatten, die Krone auf. Diese Prozesse waren jedoch gleichzeitig »nur « die Negativ-Seite neuer Wachstumstrends, die auf der Basis internationalisierter Produktion und der Peripheralisierung bestimmter Produktionsbranchen bereits seit Mitte der 70er Jahre einsetzten. Im Gegensatz zum wirtschaftlichen Wachstum der 50er und 60er Jahre (das eine breite Schicht konsumfähiger Arbeiter- und Mittelschichten mitproduzierte) gehen die gegenwärtigen Wachstumstrends einher mit der Abnahme vormals dynamischer industrieller Sektoren und Berufsgruppen. Die städtische Krise erzwang, und die neue Mobilität der Konzerne ermöglichte eine verstärkte interkommunale Konkurrenz um die Ansiedlung der Branchen und ihrer Professionals, die eine zukunftsorientierte Wachstumsperspektive ermöglichen. Resultat ist ein neuer Hierachisierungsprozeß 3 nicht nur zwischen Städten, sondern auch ein sozialräumlicher Differenzierungsprozeß innerhalb der Städte. Die gleichzeitig stattfindenden Prozesse von

- rapider Expansion hochentwickelter, unternehmensbezogener Dienstleistungen und hochtechnologischer Produktion,

- Abnahme traditioneller Aktivitäten sowohl im Dienstleistungs- als auch im Produktionsbereich, und

— der Entwicklung neuer, abgewerteter, jedoch stark expandierender ökonomischer Sektoren

polarisieren die städtische Beschäftigungs- und Einkommensstruktur. Der Bedarf an hochbezahlten technischen und professionellen Positionen (in unternehmensbezogenen Dienstleistungen genauso wie in der Produktion und Anwendung von Hochtechnologien) wächst, an traditionellen »blue and white collar jobs« dagegen besteht kein Bedarf mehr; aber die Nachfrage nach billigen, unqualifizierten, flexiblen Arbeitskräften steigt exponentiell: In den abgewerteten (aber wachsenden) Produktionsstätten (auch High Tech- und Elektronik-Industrie haben einen hohen Niedriglohnanteil, inklusive Sweatshops und »industrial homework «) und im Dienstleistungsbereich. Dieser Prozeß hat seine sozialräumliche Entsprechung in der Ausdehnung von teurer Wohnraum- und Geschäfts-Gentrifizierung einerseits und abgespaltenen, marginalisierten Communities andererseits. Besonders dort, wo die Zahl hochbezahlter Arbeitsplatzbesitzer eine kritische Masse erreicht, entstehen nicht nur die Bedingungen für die Attraktivierung der Innenstädte, d.h. sowohl bauliche 
Aufwertung als auch Reorganisation der Konsumstruktur und der urbanen Lebensstile. Diese Prozesse setzen die Verfügbarkeit eines riesigen Reservoirs von billigen Arbeitskräften voraus. Die Gentrifizierung hoher Einkommensschichten bringt also die verstärkte Nachfrage nach arbeitsintensiven Dienstleistungen mit sich, die wiederum zur Ausweitung des informellen Sektors beiträgt:

»Die Nachfrage nach billigen Arbeitskräften zur 'Bedienung' der Lebensstile der hochbezahlten Angestellten, der rapide expandierenden oberen Schichten der Professionals, ist ein Schlüsselfaktor in der Ausbreitung des informellen Sektors in Städten wie New York oder Los Angeles (Sassen-Koob 1984: 157, Übersetzung M.M.)火.

Folglich sind die Städte längst Schauplätze von Revitalisierungsschüben und Aufwertungsprozessen in den Innenstädten und umliegenden Stadtteilen geworden. Völlig separat davon, aber in struktureller gegenseitiger Abhängigkeit, reproduzieren sich die Stadtteile der Minoritäten und neuen Immigranten: ein Heer gesellschaftlicher Dienerinnen und Diener. In dieser polarisierten städtischen Struktur spielen die städtischen Bewegungen und ihre Organisationen Rollen mit jeweils unterschiedlichen Wirkungen. Die Community Organizations, die gegen Verfall und Verslumung angetreten waren, bereiteten mit ihren SelbsthilfeRenovierungsprojekten und der Aktivierung der Community durch vielfältige Projekte häufig der bald mit Macht einsetzenden Revitalisierung innerstädtischer Bereiche den Weg. Ihnen folgende Organisationen waren mehr und mehr von den »A ufwertern« getragen, den neuen professionellen Schichten und Angestellten der multinationalen Konzerne, die hier ihre Headquarters aufgeschlagen hatten. In ihren an urbaner Kultur und Lebensqualität orientierten Zielen sind sie identisch mit der dritten Strömung des Neighborhood Movement, den neuen Mittelklassen. Wo sie sich mit Enklaven alternativer Kultur und progressiver Politik vermischen, bildet sich immerhin ein Hindernis für das plan- und schrankenlos wachsende Unternehmertum. In der »zweiten Stadt« (euphemistisch »pockets of poverty « genannt), wo keine oder ungeschützte Arbeitsplätze die Regel und Informalisierung die Tendenz ist, sind die CBOs auf andere Weise ambivalent. Eingebunden in die 'Kooperation' mit Stadt/Staat und Privatunternehmen sorgen sie dafür, daß die »urban underclass« und die ausgegrenzten Stadtteile nicht völlig »abgehängt« (und unregierbar und unverfügbar) werden. Obwohl sie sich den Flexibilisierungs- und Privatisierungstendenzen kaum in den Weg stellen (oft sogar selbst an der Bereitstellung ungeschützter und schlecht entlohnter Arbeitsplätze mitwirken), bleibt die Notwendigkeit ihrer Selbstorganisation als Voraussetzung und Mittel gesellschaftlicher Steuerung doch eine permanente Paradoxie angesichts ihrer zunehmenden Marginalisierung.

In der Bundesrepublik sind Inkorporierungsprozesse sozialer Bewegungen weniger über Marktorientierung und entsprechende Entpolitisierungsprozesse gelaufen als parteienvermittelt und über Ansätze von dezentral-korporativen Strukturen (vgl. Mayer 1987). Dabei hat sich auch hier ein neues Beziehungsgeflecht zwischen Alternativgruppen, Staat und Verbänden herausgebildet. Wo der Staat mit materieller und/oder ideeller Unterstützung von Bewegungsorganisationen interveniert hat, implizierte die damit verbundene institutionelle Rahmensetzung und der Druck auf die Projekte und Interessenvertretungen, ihre Praxis als verallgemeinerungsfähige, effektive Lösungsstrategien anzubieten, ebenfalls gewisse Transformationen und Spaltungen. Zum Beispiel konnten die Instandbesetzerprojek- 
te in West Berlin der frühen 80er Jahre die Chancen ihrer Legalisierung dadurch erhöhen, daß sie kiezbezogene, kulturelle und Jugend-Projekte in ihre Arbeit und ihr Haus miteinbezogen. Sie konnten dadurch wesentlich zur Sozialintegration von »schwierigen« Kiezen beitragen und sicherten sich so die Rolle von »Vorzeigeprojekten« für Randgruppen. Gleichzeitig trat neben diese Klientel des (1982 eingerichteten) Berliner Selbsthilfe-Förderprogramms (also neben die Marginalisierten, die Zugang zu technischer Unterstützung und Beratung über IBA oder SPI hatten) eine zweite Kategorie: mittelständische und professionelle Angestellte, die als Eigentümer-(nicht Mieter-)Gemeinschaften ihr Haus renovierten. Ein Teil der Alternativgruppen wurde so zu Wegbereitern eines gehobenen urbanen Lebensstils, während andere Projekte Bindegliedfunktionen zu den aus dem ersten Arbeitsmarkt Ausgegrenzten wahrnahmen. Das in den USA beobachtete Muster von »Verarbejtung« statt Skandalisierung der neuen Armut ist auch hier bereits erkennbar. Die für die BRD-Situation spezifischen Grenzen und Widersprüche der »sozialen Experimente auf lokaler Ebene« (Evers 1986) müssen allerdings im Kontext hier stattfindender gesellschaftlicher und politischer Umstrukturierungsprozesse herausgearbeitet werden. Das US-Beispiel zeigt jedenfalls, in welche Richtung die Fragestellungen ent wickelt werden müssen, um die Chancen von »unkonventionellen lokalen Initiativen« im Rahmen der veränderten Konfliktszenarien zu bestimmen.

\section{Anmerkungen}

1 Gelder für die Entlohnung des Mitarbeiterstabs oder für Verwaltungskosten würden die Projekte nur »unselbständig« machen, lautete die allseits vorgetragene Begründung.

\section{Literatur}

Daniel Bell/Virginia Held (1969): »The Community Revolution«, in: The Public Interest, 16 (Sommer 1916), 142-177

Marc Bendick, Jr./David W. Rasmussen (1986): »Enterprise Zones and Inner-City Economic Revitalization«, in: Peterson, Lewis (Hg.), Reagan and the Cities. Washington, D. C.: The Urban Institute Press, 97-130

Peter Berger/Richard J. Neuhaus (1981): To Empower People: The Role of Mediating Structures in Public Policy. Washington, D.C.: The American Enterprise Institute

Harry C. Boyte (1980): The Backyard Revolution. Understanding the New Citizen Movements. Philadelphia: Temple UP

Harry C. Boyte (1984): Community is Possible: Repairing America's Roots. New York: Harper \& Row Stuart M. Butler (1981): Enterprise Zones: Greenlining the Inner Cities. New York, NY: Universe Books Susan E. Clarke (1984): »Neighborhood Policy Options: The Reagan Agenda«, in: Journal of the American Planners Association, 50/4 (Herbst 1984), 493-501

Gary Delgado (1986): Organizing the Movement. The Roots and Growth of ACORN. Philadelphia: Temple UP

Steven Epstein (1987): »Gay Politics, Ethnic Identity: The Limits of Social Constructionism《, in: Socialist Review, 17/3-4 (Mai-August 1987), 9-54

Adalbert Evers (1986): »Zwischen Arbeitsamt und Ehrenamt«, in: Blanke, Evers, Wollmann (Hg.): 
Die Zweite Stadt. Neue Formen lokaler Arbeits- und Sozialpolitik. Leviathan Sonderheft 7. Opladen: Westdeutscher Verlag

Joe Feagin (1985): »The Social Costs of Houston's Growth: A Sunbelt Boomtown Reexamined«, in: International Journal of Urban and Regional Research, 9, 164-185

Bob Fisher (1987): Social Movement Formation in Houston, Texas. Vortrag, Jahrestagung der DGfA, Bremen

R. Scott Fosler/Renee A. Berger (Hg.) (1982): Public-Private Partnership in American Cities. Seven Case Studies. Lexington, MA: D.C. Heath

Robert Friedman/William Schweke (1981): Expanding the Opportunity to Produce: Revitalizing the American Economy through New Enterprise Development. Washington, D. C.: The Corporation for Enterprise Development

Linda M. Gardner (1983): Community Economic Development Strategies: Creating Successful Businesses. Berkeley: National Economic Development and Law Center.

Paul K. Gatons/Michael Brintnall (1985): »Competitive Grants: The UDAG Approach $\aleph_{,}$in: R.D. Bingham, J. P. Blair (Hg.): Urban Economic Development. Beverly Hills: Sage

Norman J. Glickman (1984): »The Reagan Administration's Urban Policies«, in: Journal of the American Planners Association, 50/4 (Herbst 1984), 440-477

Andre Gorz (1985): „Die Zukunft der Arbeit. Thomas Jahn und Thomas Kluge sprechen mit Andre Gorz«, in: W. Hamman, Th. Jahn (Hg.): In Zukunft. Über den Wandel des Fortschritts. Hamburg: Rowohlt

Steve Katz (1987): Urban Restructuring and Neighborhood-Based Self-Help: The Cases of New York City and West Berlin. Ph. D. Dissertation, UCSC, Sociology

Dennis Keating (1986): »Linking Downtown Development to Broader Community Goals«, in: Journal of the American Planners Association, 52/2 (Frühjahr 1986), 133-141

Robert Kolodny (1985): Organizing for Neighborhood Development: A Community Development Guide. Washington, D.C.: Center for Community Change

Michael Lipsky (1968): »Protest as a Political Resource«, in: American Political Science Review, 62/4 (Dezember 1968), 1144-1158

Margit Mayer (1987): »Staatsknete und soziale Bewegungen«, in: Th. Kreuder, H. Loewy (Hg.): Konservativismus in der Strukturkrise. Frankfurt: Suhrkamp

Joseph McNeely (1985): »Capital, Control and Consultants: CDC Strategies for the Future«, in: Citizen Participation, 6/2 (Frühjahr 1985), 8-19

John H. Mollenkopf (1983): The Contested City. Princeton, NJ: Princeton UP

John L. Palmer/Isabel V. Sawhill (Hg.) (1984): The Reagan Record. An Assessment of America's Changing Domestic Priorities. An Urban Institute Study. Cambridge, MA: Ballinger

George E. Peterson (1984): »Federalism and the State: An Experiment in Decentralization«, in: Palmer, Sawhill (Hg.)

President's Task Force on Private Sector Initiatives (1984): Corporate Community Involvement. Washington: Partnership Data Net

Saskia Sassen-Koob (1984): „The New Labor Demand in Global Cities«, in: M. P. Smith (Hg.): Cities in Transformation. Beverly Hills: Sage

Saskia Sassen-Koob (1986): „New York City: Economic Restructuring and Immigration«, in: Development and Change, 17/1 (Januar 1986), 85-119

Mitchell Sviridoff (1982): »Neighborhood Revitalization: The Role of LISC«, in: Community Action, $1 / 3,5-8$

Avis Vidal et al. (1985): The Local Initiatives Support Corporation: Preliminary Findings of an Evaluation in Progress. Cambridge, MA: State, Local, and Intergovernmental Center, Kennedy School of Government, Harvard University

Robert L. Woodson (1982): »Helping the Poor Help Themselves«, in: Policy Review. The New Quarterly Journal of the Heritage Foundation, 21 (Sommer 1982), 73-86

Robert L. Woodson (1983): »Turning Problems into Opportunities: Utilizing Neighborhood Mediating Structures«, in: Policy Dispatch (National Center for Neighborhood Enterprise), 3 (April 1983) 